The Astrophysical Journal, 484 : L25-L28, 1997 July 20

(c) 1997. The American Astronomical Society. All rights reserved. Printed in U.S.A.

\title{
DISCOVERY OF EXTENDED BLUE HORIZONTAL BRANCHES IN TWO METAL-RICH GLOBULAR CLUSTERS ${ }^{1}$
}

\author{
R. Michael Rich, ${ }^{2}$ Craig Sosin, ${ }^{3}$ S. George Djorgovski,${ }^{4}$ Giampaolo Piotto,, 5 Ivan R. King, ${ }^{4}$ Alvio Renzini, ${ }^{6}$ E. Sterl Phinney, ${ }^{7}$ \\ Ben Dorman,, 8 James Liebert, ${ }^{10}$ AND GeORges MeYlan ${ }^{6}$ \\ Received 1997 January 13; accepted 1997 May 5
}

\begin{abstract}
We have used WFPC2 to construct $B, V$ color-magnitude diagrams of four metal-rich globular clusters, NGC 104 (47 Tuc), NGC 5927, NGC 6388, and NGC 6441. All four clusters have well populated red horizontal branches (RHB), as expected for their metallicity. However, NGC 6388 and 6441 also exhibit a prominent blue horizontal-branch (BHB) extension, including stars reaching as faint in $V$ as the turnoff luminosity. This discovery demonstrates directly for the first time that a major population of hot horizontal-branch (HB) stars can exist in old, metal-rich systems. This may have important implications for the interpretation of the integrated spectra of elliptical galaxies.

The cause of the phenomenon remains uncertain. We examine the possibility that NGC 6388 and 6441 are older than the other clusters, but a simple difference in age may not be sufficient to produce the observed distributions along the HB. The high central densities in NGC 6388 and 6441 suggest that the existence of the BHB tails might be caused by stellar interactions in the dense cores of these clusters, which we calculate to have two of the highest collision rates among globular clusters in the Galaxy. Tidal collisions might act in various ways to enhance loss of envelope mass and therefore populate the blue side of the HB. However, the relative frequency of tidal collisions does not seem large enough (compared to that of the clusters with pure RHBs) to account for such a drastic difference in HB morphology. While a combination of an age difference and dynamical interactions may help, prima facie the lack of a radial gradient in the BHB/RHB star ratio seems to argue against dynamical effects playing a role.

Subject headings: Galaxy: kinematics and dynamics - globular clusters: individual (NGC 104, 47 Tucanae, NGC 5927, NGC 6388, NGC 6441) — stars: horizontal-branch
\end{abstract}

\section{INTRODUCTION}

It is well known that a primary strong correlation exists between the color distribution of the horizontal-branch (HB) stars in Galactic globular clusters and their metallicities: metal-poor clusters have essentially blue HBs (BHBs) while metal-rich clusters have stubby red HBs (RHBs). Hot HB stars arise as a result of mass loss in red giant branch (RGB) stars, and any process that reduces the mean envelope mass either before or during the $\mathrm{HB}$ phase can also give rise to a $\mathrm{BHB}$. For example, high metallicity may promote a hot extension of the $\mathrm{HB}$ in very old stellar populations if mass loss increases with metallicity (see Greggio \& Renzini 1990). A higher age for the cluster will reduce both the main-sequence turnoff and the $\mathrm{HB}$

${ }^{1}$ Based on observations with the NASA/ESA Hubble Space Telescope, obtained at the Space Telescope Science Institute, which is operated by AURA, Inc., under NASA contract NAS 5-26555.

2 Department of Astronomy, Columbia University, Mail Code 5242, New York, New York 10027; rmr@astro.columbia.edu.

${ }^{3}$ University of California, Department of Astronomy, 601 Campbell Hall, Berkeley, CA 94720-3411; csosin@aster.berkeley.edu, king@glob.berkeley. edu.

${ }^{4}$ Department of Astronomy, California Institute of Technology, MS 105-24, Pasadena, CA 91125; george@astro.caltech.edu.

5 Dipartimento di Astronomia, Università di Padova, Vicolo dell' Osservatorio 5, I-35122 Padova, Italy; piotto@astrpd.pd.astro.it.

${ }^{6}$ European Southern Observatory, Karl-Schwarzschild-Strasse 2, Garching bei München, Germany; arenzini@eso.org, gmeylan@eso.org.

7 Theoretical Astrophysics, MS 130-33, California Institute of Technology, Pasadena, CA 91125; esp@tapir.caltech.edu.

${ }^{8}$ Laboratory for Astronomy and Solar Physics, Code 681, NASA/Goddard Space Flight Center, Greenbelt, MD 20771; dorman@parfait.gsfc.nasa.gov.

${ }_{9}$ Astronomy Department, University of Virginia, Charlottesville.

10 Steward Observatory, University of Arizona, Tucson, AZ 85721; liebert@ as.arizona.edu. mass, thus making the production of BHB stars more likely. Also, dynamical interactions in dense clusters might favor the production of BHB stars (see Fusi Pecci et al. 1993). The first option may be related to the increase in the UV flux toward shorter wavelengths for $\lambda \lesssim 2000 \AA$ in some elliptical galaxies (Code \& Welch 1979; Bertola et al. 1980). The hot stars responsible for the UV rising flux may indeed be HB stars that have somehow managed to lose a major fraction of their envelopes, thus spending their HB lifetime at high effective temperatures (Greggio \& Renzini 1990). While there is some circumstantial evidence to support this idea (e.g., Ferguson \& Davidsen 1993; Dorman, O'Connell, \& Rood 1995), the actual presence of a significant proportion of hot HB stars in an old and metal-rich population had not been observed to date.

In all such populations where individual stars are resolved, the HB stars are indeed grouped in a red clump. For example, Hubble Space Telescope (HST) observations of the old metalrich clusters NGC 6528 and NGC 6553 have shown that these bulge clusters have a purely RHB (Ortolani et al. 1995). However, these two clusters might not be fully representative of all possible varieties of metal-rich populations: Rich, Minniti, \& Liebert (1993) obtained IUE spectra of a sample of metal-rich $([\mathrm{Fe} / \mathrm{H}]>-0.6)$ clusters including NGC 6637, 6441, and 6388, finding weak but significant UV fluxes. Also noteworthy is the discovery by Kaluzny \& Udalski (1992) and Liebert, Saffer, \& Green (1994) of hot HB stars in the old open cluster NGC 6791, which is considerably more metal-rich at $[\mathrm{Fe} / \mathrm{H}]=+0.23$, although it is younger and lower in stellar density compared with the globular clusters.

Some globular clusters have a bimodal HB temperature distribution, with a separate group of very hot HB stars coexisting with a cooler $\mathrm{HB}$ component. The first such cases to 
TABLE 1

Relevant Physical Parameters of the Clusters in this Study

\begin{tabular}{|c|c|c|c|c|c|c|c|c|}
\hline $\begin{array}{l}\text { NGC } \\
(1)\end{array}$ & $\begin{array}{c}{[\mathrm{Fe} / \mathrm{H}]} \\
(2)\end{array}$ & $\begin{array}{l}M_{V} \\
(3)\end{array}$ & $\begin{array}{c}(m-M)_{0} \\
(4)\end{array}$ & $\begin{array}{l}\log r_{\mathrm{c}} \\
(\mathrm{pc}) \\
(5)\end{array}$ & $\begin{array}{c}\mu_{0, V} \\
\left(\mathrm{mag}_{(6)} \operatorname{arcsec}^{-2}\right) \\
(6)\end{array}$ & $\begin{array}{c}\sigma_{\mathrm{obs}} \\
\left(\mathrm{km} \mathrm{s}^{-1}\right) \\
(7)\end{array}$ & $\begin{array}{c}N_{\text {coll }} \\
\text { (total) } \\
(8)\end{array}$ & $\begin{array}{c}N_{\text {coll }} \\
\text { (our field) } \\
(9)\end{array}$ \\
\hline $104 \ldots \ldots$ & -0.71 & -9.42 & 13.31 & -0.56 & 14.30 & 9.8 & 1.00 & 1.00 \\
\hline $5927 \ldots \ldots$ & -0.31 & -7.94 & 14.52 & -0.01 & 15.48 & $\ldots$ & 0.37 & 0.05 \\
\hline $6388 \ldots \ldots$ & -0.60 & -9.46 & 15.21 & -0.40 & 13.45 & 18.9 & 3.89 & 2.41 \\
\hline $6441 \ldots \ldots$ & -0.53 & -9.30 & 15.15 & -0.46 & 13.53 & 17.6 & 3.29 & 3.12 \\
\hline $6528 \ldots \ldots$ & -0.23 & -6.44 & 14.10 & -0.78 & 14.70 & $\ldots$ & 0.45 & $\ldots$ \\
\hline $6553 \ldots \ldots$ & -0.29 & -7.56 & 12.72 & -0.25 & 15.04 & $\ldots$ & 0.52 & $\ldots$ \\
\hline $6624 \ldots \ldots$ & -0.37 & -7.82 & 14.54 & -0.82 & 14.45 & 5.3 & 0.61 & 0.85 \\
\hline
\end{tabular}

NoTE.-All data in this table are from Djorgovski 1993 and Pryor \& Meylan 1993, except for the collision rates and the core radius of 47 Tuc (NGC 104). For the latter we used the value given by De Marchi \& Paresce 1996. The collision rates in col. (8) are calculated using the formula (King 1997) given in the text and are normalized

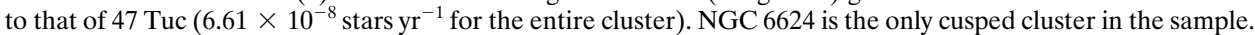

be noticed included NGC 1851 and NGC 2808. These clusters have exceptionally high central stellar densities, which suggested the possibility of a dynamical origin of the BHB stars in these clusters, as extra mass loss during the RGB phase may be promoted by tidal encounters (Renzini 1983, 1984). Some of these clusters (e.g., M15) have color-magnitude diagrams (CMDs) that are identical to those of other low-density clusters of the same metallicity, except for the addition of a hot $\mathrm{HB}$ component that is absent in the low-density clusters (Buonanno, Corsi, \& Fusi Pecci 1985). Fusi Pecci et al. (1993) found a correlation between cluster density and the presence of a hot HB population and its extension, in particular for the intermediate-metallicity clusters, but their sample did not include clusters with $[\mathrm{Fe} / \mathrm{H}]>-0.6$. Djorgovski et al. (1991) measured radial color gradients in some high-concentration clusters, which in some (but perhaps not all) cases could be traced to the gradients in the ratio of BHB to RGB stars, thus strengthening the case for a possible connection between the presence of hot HB stars and the dynamical state of the clusters. There is, on the whole, a growing body of evidence that cluster dynamics, through close stellar encounters and/or formation of binaries, can significantly alter the stellar populations in dense stellar systems; for a review and further references, see Djorgovski \& Piotto (1993).

In this Letter we report the discovery of a BHB extension in the metal-rich globular clusters NGC 6388 and NGC 6441, using the WFPC2 on board the HST.

\section{OBSERVATIONS AND COLOR-MAGNITUDE DIAGRAMS}

The relevant properties of the program clusters are given in Table 1, together with those of four additional metal-rich clusters also observed with the refurbished HST but not included in the present program. Most of the data in the first six columns are from the compilation by Djorgovski (1993). All the listed clusters have quite similar characteristics, except that NGC 6388 and 6441 have high velocity dispersions $\left(18 \mathrm{~km} \mathrm{~s}^{-1}\right.$; Pryor \& Meylan 1993) and very high central surface brightnesses. The cluster metallicities have been determined from the Ca II triplet method of Armandroff \& Zinn (1988) to be $[\mathrm{Fe} / \mathrm{H}]=-0.60 \pm 0.15$ for NGC 6388 and $-0.53 \pm 0.11$ for NGC 6441.

As part of a survey of globular cluster cores, NGC 6441 and NGC 6388 were observed by the WFPC2 on the HST on 1995 September 12 and on 1996 February 25, respectively. Several exposures were taken through the F555W and F439W filters, which approximate Johnson $V$ and $B$. These exposures were planned to reach slightly fainter than the main-sequence turnoff; total integration times were $62 \mathrm{~s}$ (NGC 6388) and $64 \mathrm{~s}$ (NGC 6441) in F555W, and $370 \mathrm{~s}$ in F439W for both clusters. Longer exposures were taken using the F218W filter. We will report on the analysis of the UV data at a later time.

The images were processed by the standard HST pipeline and were then trimmed, cleaned of cosmic rays, and combined using standard IRAF tasks. We then used DAOPHOT (Stetson 1987) to identify stars and to measure magnitudes using standard PSF-fitting techniques. More details of the reduction procedure for all clusters observed in our HST program will be presented in forthcoming papers. The photometry was calibrated to the WFPC2 instrumental system using the zero points given by Holtzman et al. (1995). The F439W and F555W magnitudes were then converted to Johnson $B$ and $V$, using transformations given in that same paper.

Figure 1 shows the color-magnitude diagrams of NGC 6388 and NGC 6441 compared with those of 47 Tuc and NGC 5927. While the two latter clusters have only the classic RHBs typical of metal-rich globular clusters, NGC 6388 and 6441 have additional extended $\mathrm{BHBs}$. The two $\mathrm{BHBs}$ have identical ridge lines when the CMDs are shifted to identical distance modulus and reddening.

The temperatures reached by the BHB stars are so high that their $V$-band magnitudes are determined by the large bolometric corrections (several magnitudes), and the BHB becomes a vertical branch in these CMDs, reaching below the main-sequence turnoff. Note that a hint of a blueward extension of the HB of NGC 6388 was present already in a ground-based CMD of this cluster (Silbermann et al. 1994) but that photometry was too shallow to reach even the bright top of the vertical branch. Nevertheless, this suggests that the blue extension may be present at even larger distances from the cluster center than those explored by our WFPC2 frames.

Other metal-rich globular clusters known to have $H S T$ CMDs include NGC 6528 and 6553 (Ortolani et al. 1995) and NGC 6624 (Sosin \& King 1995). All these clusters have RHBs similar to that of 47 Tuc and NGC 5927. Hence, NGC 6388 and NGC 6441 stand out as the only metal-rich clusters with prominent BHB populations. Since their metallicities are comparable to those of the other clusters, with normal RHBs, we are forced to conclude that this is yet another manifestation of a second-parameter effect, by which we mean that metallicity is not the only factor that governs the morphology of the HB. 

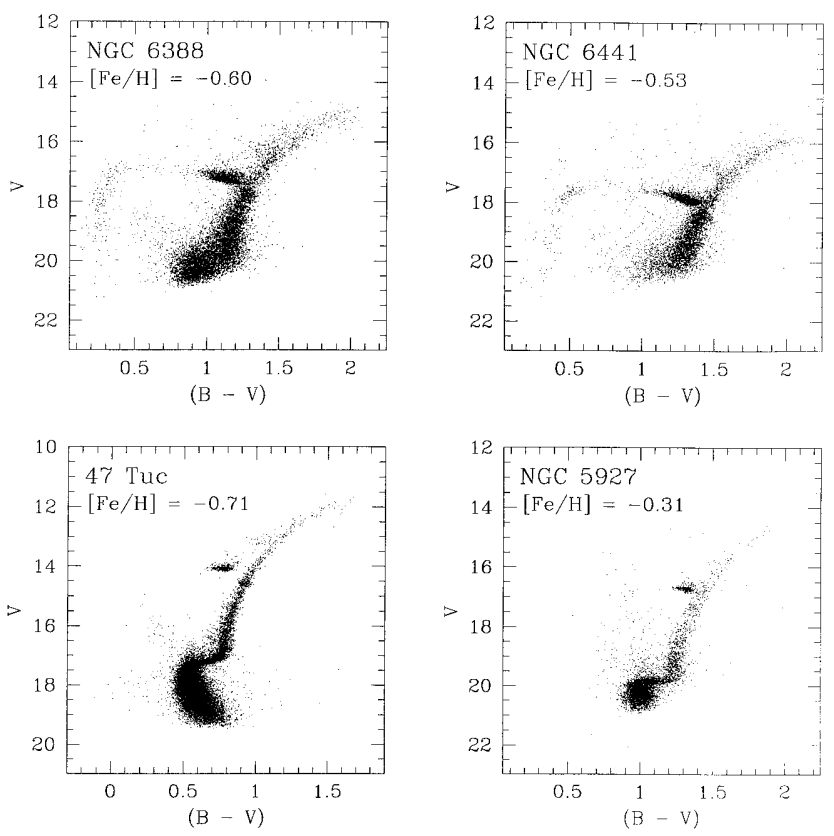

FIG. 1.-Color-magnitude diagrams constructed from F439W $(B)$ and F555W $(V)$ WFPC2 images of the globular clusters in this study. The red stubby horizontal branches of 47 Tuc and NGC 5927 are typical for clusters of such high metallicity $([\mathrm{Fe} / \mathrm{H}]>-0.8)$. Extended blue horizontal branches (such as those in NGC 6388 and 6441) have never before been seen in such metal-rich globular clusters; they are the only two cases now known. Notice also that the morphology of the red horizontal branches of NGC 6388 and 6441 is unusual compared to the other clusters.

\section{DISCUSSION}

The presence of the classical second-parameter effect, i.e., a mostly red $\mathrm{HB}$ in a metal-poor cluster, is well established among clusters in the outer halo. However, Lee, Demarque, \& Zinn (1994) found no evidence for a second-parameter effect among clusters with Galactocentric distance less than about 8 kpc. Our findings clearly demonstrate that second-parameter effects are actually present among Galactic bulge globular clusters, although the physical origin of the effect may be different in the bulge and in the halo. Given that the clusters in Table 1 with and without BHB extensions do not differ substantially in their metallicities, we consider whether either age differences or stellar interactions can be responsible for the extended HBs in NGC 6388 and 6441.

The effective temperature of Population II stars burning helium in their cores is primarily a function of how much envelope has survived mass loss on the RGB; see, for example, the extensive grids of HB models by Sweigart \& Gross (1976) or Dorman, Rood, \& O'Connell (1993). In principle, all stellar populations develop a BHB when they become sufficiently old, as stars with lower and lower mass evolve off the main sequence and therefore reach the $\mathrm{HB}$ with a lower and lower envelope mass. However, the age at which this happens is a function of metallicity, helium enrichment, and mass loss during the RGB phase (e.g., Greggio \& Renzini 1990). In turn, mass loss likely depends on composition, but we have neither an observational nor a theoretical relationship for this dependence, which prevents a priori predictions of the HB morphology.

At high metallicity, an increase in age can thus populate a blue tail with stars at the low end of the mass distribution (see
Renzini 1977, Fig. 3.1) while the dominant HB remains red. At the composition $[\mathrm{Fe} / \mathrm{H}]=-0.47,[\mathrm{O} / \mathrm{Fe}]=+0.23$, the nearest in the Dorman et al. (1993) model grid, the difference in envelope mass between ZAHB stars at $5000 \mathrm{~K}$ and $11,500 \mathrm{~K}$ (approximately the difference in $T_{\text {eff }}$ between stars on the red and blue sides of the HB) is only $0.04 M_{\odot}$, which would correspond to an age difference of about $4 \mathrm{Gyr}$, the two clusters with hot HB extensions being older. However, it is difficult to prove whether such an age difference alone can transform a stubby red HB such as that of 47 Tuc into a HB such as that exhibited by NGC 6388 or 6441 . There is indeed a hint for a mildly bimodal distribution of stars on the HBs of these two clusters (see Fig. 1), which may require a bimodal distribution of HB masses. Such bimodality is not required to account for the HB of 47 Tuc, although it may be hidden by the very weak dependence of ZAHB temperature on envelope mass for red HB stars. We conclude that further study is required to assess whether an older age can account for the peculiar HBs of NGC 6388 and 6441. If these clusters are indeed older, then they would also have redder, fainter turnoffs, but deeper HST observations are needed for this test.

We now turn to a second option, i.e., to the possible dynamical origin of the hot HB component in these clusters. Again, the average extra mass loss caused by environmental effects must exceed about $0.04 M_{\odot}$ in order to generate blue HBs such as those shown in Figure 1. Tidal stripping of part of the envelope while stars are ascending the RGB is one possibility. However, other more complicated alternatives can be envisaged besides direct stripping at the time of the tidal collision. For example, orbital angular momentum can be transferred in a close flyby and stored in a red giant envelope. In turn, this might promote enhanced mass loss along the remaining fraction of the RGB phase or even deep mixing between the envelope and the upper part of the hydrogenburning shell (Sweigart 1997a, 1997b). Unfortunately, hydrodynamical phenomena such as red giant mass loss and mixing are exceedingly difficult to model; hence, these possibilities remain conjectural at this stage.

There are, however, established correlations between the blue extent of the HB and cluster density or concentration. Fusi Pecci et al. (1993) find the strongest correlation of their blue tail (BT) length parameter with $c-2 M_{V}$, where $c$ is the King cluster concentration parameter and $M_{V}$ is the cluster integrated absolute magnitude. However, while $c-2 M_{\mathrm{V}}=$ 20.8 and 20.6 for NGC 6388 and 6441, respectively, $c-$ $2 M_{V}=21.0$ in the pure-RHB cluster 47 Tuc. Still, we note that NGC 6388 and 6441 stand out as having some of the highest values of the central surface brightness and velocity dispersion among globular clusters in the Galaxy (see Pryor \& Meylan 1993; Djorgovski 1993; see also Table 1), indicating very high central stellar densities and hence high rates of stellar interactions. It is of further interest to note that the globular cluster G1 (= Mayall II) in M31 has a modest blueward HB extension to its dominant RHB (Rich et al. 1996). This cluster has a central surface brightness as high as that of NGC 6388 and 6441 and a metallicity comparable to that of 47 Tuc.

It is reasonable to assume that in a cluster the frequency of very close encounters is some multiple of the frequency of stellar physical collisions, for which early estimates were made by Hills \& Day (1976). King (1997) shows that the rate of stellar collisions in a King model globular cluster is about $5 \times$ $10^{-15}\left(\Sigma_{0}^{3} r_{c}\right)^{1 / 2}$, where $\Sigma_{0}$ is the central surface brightness in the units of $L_{\odot V} \mathrm{pc}^{-2}$ (equivalent to $\mu_{V}=26.41$ ), and $r_{c}$ is the core 
radius in pc. (This formula was derived by integrating the local collision rate over a density distribution that is a good approximation for King models. It is still approximate, however, in that all stellar masses were taken to be $0.4 M_{\odot}$ and all stellar radii $0.5 R_{\odot}$.)

Column (9) gives the total number of the stars appearing in our WFPC2 fields that should have undergone a collision, relative to 47 Tuc. We obtained these values by dividing the numbers in column (8) by the luminosity of the core of each cluster, and then multiplying by the number of giants in our WFPC2 image of that cluster. It appears that NGC 6388 and 6441 again stand out with the highest relative collision rates, but the difference with respect to the other clusters seems too small to account for the large difference in HB morphology. In NGC 6388, for example, we see about 180 BHB stars, and one would therefore expect to see about 75 of them in 47 Tuc, but we see none in Figure 1. Therefore, it appears difficult for dynamical processes alone to account for the observed effect. Quite possibly, besides dynamics another factor is at work, for example, age. Perhaps tidally stripped stars exist also in 47 Tuc, but the cluster is not old enough for them to have started to populate the BHB.

Another problem for the close encounter hypothesis arises from considering the radial distributions of the BHB stars. Since most tidal encounters will take place within the dense core of the cluster, one would expect the BHB stars to be more centrally concentrated than the RHB stars. However, neither in NGC 6388 nor in NGC 6441 is such an effect present: we find that $\mathrm{BHB}$ and $\mathrm{RHB}$ stars have just the same radial distribution within the statistical uncertainty. The only group of stars with a markedly different radial distribution is the (centrally concentrated) blue stragglers.

It might be argued that dynamical relaxation will quickly cause the encounter products to diffuse out of the cluster center, thus smoothing out their distribution. To test this hypothesis, we performed Fokker-Planck simulations of the evolution of stellar orbits in model clusters with the parame- ters of NGC 6388 and NGC 6441. These show that $10^{8} \mathrm{yr}$ and $10^{9} \mathrm{yr}$ after visiting the cluster core, stripped stars will still be concentrated within $2 r_{c}$ and $4 r_{c}$, respectively. We find instead the $\mathrm{BHB} / \mathrm{RHB}$ ratio to be flat out to at least about $6 r_{c}$. Stripping stars at an earlier stage, while still on the main sequence, would not help, as this will only lengthen their lifetimes rather than produce core helium-burning stars with less massive envelopes.

A number of scenarios have been suggested to make BHB stars from binaries (Bailyn 1995), but these would also predict a central concentration of the BHB stars that is not present in our data. Nonetheless, a binary related origin remains an intriguing possibility. Green, Saffer, \& Liebert (1997) find evidence that some of the BHB stars in the old open cluster NGC 6791 are radial velocity variables, i.e., presumably binaries. Similarly, the field subdwarf B stars show a very high frequency of binaries (Allard et al. 1994).

In conclusion, we still lack a definitive explanation for the peculiar HBs in NGC 6388 and 6441. There is a hint that dynamical effects, perhaps along with an age difference, are at work. If stellar encounters play a role in causing these peculiar HBs, then other clusters of comparable surface brightness should also exhibit the same peculiarity to some extent. These may include HP 1, Liller 1, and NGC 6440, which all have very high surface brightness, and future $H S T$ imaging of them can test this hypothesis. Whatever their cause, the existence of hot $\mathrm{HB}$ extensions in metal-rich stellar populations will have broad implications for our understanding of the evolution of globular cluster stars and could affect our interpretation of the spectra of other old stellar systems, such as elliptical galaxies.

This work was supported in part by NASA grant GO-6095 from STScI (R. M. R., S. G. D., I. R. K., C. S.), NASA grant NAG5-2756 (E. S. P.), NASA grants NAG5-700 and NAGW4106 (B. D.), the Bressler Foundation (S. G. D.), and Agenzia Spaziale Italiana (G. P., A. R.).

\section{REFERENCES}

Allard, F., Wesemael, F., Fontaine, G., Bergeron, P., \& Lamontagne, R. 1994 AJ, 107, 1565

Armandroff, X., \& Zinn, X. 1988, AJ, 96, 92

Bailyn, C. D. 1995, ARA\&A, 33, 133

Bertola, F., Capaccioli, M., Holm, A. V., \& Oke, J. B. 1980, ApJ, 237, L65

Buonanno, R., Corsi, C. E., \& Fusi Pecci, F. 1985, A\&A, 145, 97

Code, A. D., \& Welch, G. A. 1979, ApJ, 228, 95

De Marchi, G., \& Paresce, F. 1996, ApJ, 468, L51

Djorgovski, S. 1993, in ASP Conf. Proc. 50, Structure and Dynamics of Globular Clusters, ed. S. G. Djorgovski \& G. Meylan (San Francisco: ASP), 373

Djorgovski, S., \& Piotto, G. 1993, in ASP Conf. Proc. 50, Structure and Dynamics of Globular Clusters, ed. S. G. Djorgovski \& G. Meylan (San Francisco: ASP), 203

Djorgovski, S., Piotto, G., Phinney, E. S., \& Chernoff, D. F. 1991, ApJ, 372, L41

Dorman, B., Rood, R. T., \& O'Connell, R. W. 1993, ApJ, 419, 592

Dorman, B., O'Connell, R. W., \& Rood, R. T. 1995, ApJ, 442, 105

Ferguson, H., \& Davidsen, A. 1993, ApJ, 408, 92

Fusi Pecci, F., Ferraro, F. R., Bellazzini, M., Djorgovski, S., Piotto, G., \& Buonanno, R. 1993, AJ, 105, 1145

Green, E. M., Saffer, R. A., \& Liebert, J. W. 1997, in 3d Conf. on Faint Blue

Stars, ed. A. G. D. Philip, J. W. Liebert, \& R. A. Saffer (Schenectady: L. Davis), in press

Greggio, L., \& Renzini, A. 1990, ApJ, 364, 35

Hills, J. G., \& Day, C. A. 1976, Astrophys. Lett., 17, 87
Holtzman, J. A., et al. 1995, PASP, 107, 106

Kaluzny, J., \& Udalski, A. 1992, Acta Astron., 42, 29

King, I. R. 1997, Introduction to Stellar Dynamics (Mill Valley: University Science), in press

Lee, Y.-W., Demarque, P., \& Zinn, R. J. 1994, ApJ, 423, 248

Liebert, J., Saffer, R. A., \& Green, E. M. 1994, AJ, 107, 1408

Ortolani, S., et al. 1995, Nature, 377, 701

Pryor, C., \& Meylan, G. 1993, in ASP Conf. Proc. 50, Structure and Dynamics of Globular Clusters, ed. S. G. Djorgovski \& G. Meylan (San Francisco: ASP), 357

Renzini, A. 1977, in Advanced Stages in Stellar Evolution, ed. P. Bouvier \& A. Maeder (Sauverny: Geneva Obs.), 151

1983, Mem. Soc. Astron. Italiana, 54, 335

1984, in Observational Tests of Stellar Evolution Theory, ed. A. Maeder \& A. Renzini (Dordrecht: Kluwer), 21

Rich, R. M., Mighell, K. J., Freedman, W. L., \& Neill, J. D. 1996, AJ, 111, 768

Rich, R. M., Minniti, D., \& Liebert, J. 1993, ApJ, 406, 489

Silbermann, N. A., Smith, H. A., Bolte, M., \& Hazen, M. L. 1994, AJ, 107, 1764

Sosin, C., \& King, I. R. 1995, AJ, 109, 639

Stetson, P. B. 1987, PASP, 99, 191

Sweigart, A. V. 1997a, ApJ, 474, L23

. 1997b, in 3d Conf. on Faint Blue Stars, ed. A. G. D. Philip, J. W. Liebert, \& R. A. Saffer (Schenectady: L. Davis), in press Sweigart, A. V., \& Gross, P. G. 1976, ApJS, 32, 367 